\title{
Genetic and Virulence Diversity in Verticillium dahliae Populations Infecting Artichoke in Eastern-Central Spain
}

\author{
Rafael M. Jiménez-Díaz, Jesús Mercado-Blanco, Concepción Olivares-García, \\ Melania Collado-Romero, José Bejarano-Alcázar, Dolores Rodríguez-Jurado, \\ Ana Giménez-Jaime, José García-Jiménez, and Josep Armengol
}

First and third authors: Departamento de Agronomía, Escuela Técnica Superior de Ingenieros Agrónomos y Montes, Universidad de Córdoba, and Instituto de Agricultura Sostenible (IAS), Consejo Superior de Investigaciones Científicas (CSIC), Apartado 4084, 14080 Córdoba, Spain; second and fourth authors: IAS-CSIC; fifth and sixth authors: CIFA Alameda del Obispo, IFAPA, Junta de Andalucía, Apartado 3092, 14080 Córdoba, Spain; and seventh, eighth, and ninth authors: Instituto Agroforestal Mediterráneo, Universidad Politécnica de Valencia, Valencia, Camino de Vera s/n, 46022 Valencia, Spain.

Accepted for publication 2 November 2005.

\begin{abstract}
Jiménez-Díaz, R. M., Mercado-Blanco, J., Olivares-García, C., ColladoRomero, M., Bejarano-Alcázar, J., Rodríguez-Jurado, D., GiménezJaime, A., García-Jiménez, J., and Armengol, J. 2006. Genetic and virulence diversity in Verticillium dahliae populations infecting artichoke in eastern-central Spain. Phytopathology 96:288-298.

Severe Verticillium dahliae attacks have occurred in artichoke crops in the Comunidad Valenciana region of eastern-central Spain since the late 1990s. Knowledge of genetic and virulence diversity in the pathogen population is a key factor for the management of the disease through disease risk assessment as well as development and use of resistant cultivars. $V$. dahliae isolates from artichoke (109 isolates) and cotton (three isolates) in that region were characterized by vegetative compatibility grouping (VCG), and specific polymerase chain reaction assays using three sets of primer pairs that differentiate the cotton-defoliating (D) and -nondefoliating (ND) V. dahliae pathotypes. In all, 35 and $39 \mathrm{~V}$. dahliae isolates representative of the identified VCGs and geographic origins were tested for virulence to artichoke cvs. Nun 6374 and Nun 9444, and cotton cv.

(one isolate), VCG2A (31 isolates), VCG2B (72 isolates), and VCG4B (three isolates). The three cotton isolates were VCG1A. Isolates in VCG2B were distributed across the region and were the most prevalent isolates in the northern part. Conversely, $83.9 \%$ of isolates in VCG2A were recovered from the southern part of the region. Two subgroups of isolates were identified in VCG2B based on heterokaryon compatibility with either international or local tester isolates, which further showed diversity in the amplification of 334- and 824-bp DNA fragments which are markers of the D and ND pathotypes, respectively. Virulence of isolates to artichoke and cotton correlated with VCG but the pattern of correlation varied with the host. VCG1A isolates from artichoke and cotton induced defoliation in cotton but not in artichoke. Collectively, isolates of VCG2B and VCG4B were the most virulent and isolates of VCG1A or HSI were the least virulent to artichoke; but isolates of VCG1A were more virulent to cotton than those of any other VCG. Also, molecular subgrouping in VCG2B determined by amplification of the 334- and 824-bp markers correlated with virulence of isolates to the two hosts tested.
\end{abstract} Acala SJ-2, respectively. Four VCGs were identified among 107 artichoke isolates, and 2 isolates were heterokaryon self-incompatible: VCG1A
Additional keywords: Gossypium hirsutum, molecular marker.
Knowledge of genetic and virulence diversity in plant pathogen populations is key for efficient plant disease management. For Verticillium dahliae Kleb., the agent of Verticillium wilt diseases of hundreds of herbaceous and woody crops (45), inability to recognize the genetic diversity within the pathogen has limited our understanding of disease development and efficacy of management practices (49). During the last decade, studies using vegetative compatibility and molecular analyses have improved our understanding of $V$. dahliae and led to the realization that genetic diversity in this pathogen is higher than previously thought $(2,34,49)$.

Vegetative compatibility refers to the genetically controlled ability of individual fungal strains to undergo hyphal anastomosis and form stable heterokaryons. Vegetatively compatible isolates of a fungal species are placed in the same vegetative compatibility group (VCG). Because V. dahliae is a strictly asexually reproducing fungus $(30,45)$, vegetative compatibility is a prerequisite to genetic exchange among different strains of the pathogen. Therefore, V. dahliae isolates in different VCGs are thought to be

Corresponding author: R. M. Jiménez-Díaz: E-mail address: ag1jidir@uco.es

DOI: 10.1094/PHYTO-96-0288

(C) 2006 The American Phytopathological Society genetically isolated populations each having the potential to share a common gene pool which, among them, may vary in many characteristics, including those related to pathogenicity and virulence $(34,49)$. VCGs are identified using spontaneous nitratenonutilizing (nit), auxotrophic mutants that show a thin but expansive growth on minimal medium with nitrate as a sole nitrogen source. Isolates are considered vegetatively compatible when complementing nit mutants anastomose and produce wild-type growth. Using this approach, VCG1, VCG2, VCG3, and VCG4 were identified among V. dahliae isolates from diverse geographic and plant sources worldwide $(7,14,15,21,32,33,37,39,52,53,58)$. Recently, a new VCG, VCG6, was identified among V. dahliae isolates from pepper (Capsicum annuиm L.) in California (3). Based on the frequency and vigor of complementation, VCG1, VCG2, and VCG4 each was further divided into two subgroups designated $\mathrm{A}$ and $\mathrm{B}(7,33,52)$. A set of isolates and nit mutant testers were described that represents the subgroups of those three VCGs $(32,33,52,53)$. The use of this set in VCG studies would help in establishing an internationally uniform system for classification and exchange of information about VCGs in $V$. dahliae $(34,49)$.

Vegetative compatibility in $V$. dahliae populations has been studied according to two major approaches (i.e., using collections of isolates from diverse geographic origin and source plants, or 
concentrating on populations occurring in particular geographic regions or specific crops). The first of those approaches has helped to estimate that limited VCG diversity exists in V. dahliae, and showed that VCG1, VCG2, and VCG4 occur worldwide $(34,49)$. The few studies about VCGs associated with specific crops indicate that some VCGs may prevail on a crop in a given geographic area $(3,25,32,33,52,53)$, and that VCG and virulence (i.e., the amount of disease caused in a host plant genotype) of $V$. dahliae isolates from a given crop may be correlated $(33,37$, 39,53). The extent of VCG association and differential virulence of $V$. dahliae in major crops merits further studies because it would be valuable for disease prediction and breeding for resistance (34).

Although VCGs may correspond to distinct gene pools within a fungal species, isolates in $V$. dahliae and other strictly asexually reproducing fungi such as Fusarium oxysporum have shown some degree of vegetative compatibility with otherwise distinct VCGs (designated as "bridging isolates") $(31,32,35)$. That opens the possibility that some gene flow might occur between VCGs which is relevant to the emergence of new virulence. Molecular studies would help in assessing the genetic variation that might exist within and among VCGs. However, information relating VCGs with molecular variability is scarce and molecular grouping may or may not correlate with VC grouping $(3,23,24,34)$. In previous studies for the molecular characterization of cotton-defoliating (D, belonging to VCG1A) and -nondefoliating (ND, belonging to either VCG2A or VCG4B) V. dahliae isolates from Spain, we developed specific primers and identified molecular markers that differentiate between the D and ND pathotypes in specific polymerase chain reaction (PCR) analyses $(39,41-43,46)$. However, later use of those primers and markers with a larger and more diverse collection of isolates resulted in some inconsistencies in the production of the marker amplicons (19), suggesting molecular diversity among isolates within a VCG.

Verticillium wilt of artichoke (Cynara cardunculus L. var. scolymus), caused by $V$. dahliae, is of increasing concern in artichoke production. Severe outbreaks of this disease were reported in France in the 1960s (13), Italy and Greece in the 1980s $(18,55)$, and California in the 1990s (4). In Spain, severe attacks by the disease were reported to occur since the late 1990s in the Comunidad Valencian region of eastern-central Spain, the main artichoke production area in the country (1). Use of pathogen-free planting material in noninfested soil (18), as well as soil fumigation (16) and soil solarization (55), can control the disease; however, use of resistant cultivars would be more economically efficient and sustainable (17). There was no information available about variation in virulence among $V$. dahliae isolates from artichoke; a VCG2 isolate from California was highly virulent to most of the 14 tested plant species (5). A better understanding of genetic and virulence diversity among host $V$. dahliae isolates would contribute to the efficient management of Verticillium wilt of artichoke, in particular through development of resistant cultivars.

The main objectives of this study were to (i) estimate the spectrum of VCG diversity among $V$. dahliae isolates from artichoke in eastern-central Spain; (ii) reveal any association among VCG, molecular markers, and virulence to artichoke and cotton cultivars; and (iii) assess the validity of molecular markers designed to differentiate D and ND $V$. dahliae pathotypes for the characterization of $V$. dahliae isolates from artichoke.

\section{MATERIALS AND METHODS}

Fungal isolates and culture conditions. A collection of 112 $V$. dahliae isolates from artichoke (109 isolates) and cotton (3 isolates) was studied (Table 1). Isolates from artichoke were obtained in 2001 and 2002 from 70 wilt-affected crops surveyed in Alicante (34 fields), Castellón (14 fields), and Valencia (22 fields) provinces of the Comunidad Valenciana region, which grows over 6,000 ha of artichoke (Fig. 1). Two $V$. dahliae isolates were obtained from each of 17,8 , and 14 fields sampled in Alicante, Castellón, and Valencia, respectively, and one from each of the remaining 31 fields. The cropping history was recorded for six sampled fields in Alicante, eight in Castellón, and four in Valencia. Isolates were single spored, and two monoconidial cultures per isolate were arbitrarily chosen for VCG characterization. The identities of all single-spore isolates were determined on the basis of conidiophores and microsclerotia formed on plum-lactoseyeast extract agar (PLYA) (54) and further confirmed by speciesspecific PCR assays using primers DB19 and DB22 (12). Isolates were stored by covering cultures on PLYA with sterile liquid paraffin (8) at $4^{\circ} \mathrm{C}$ in the dark. Active cultures of isolates were obtained on water agar and further subculturing on Czapek-Dox agar (CDA) (Difco Laboratories, Detroit, MI) at $24^{\circ} \mathrm{C}$ in the dark. For inoculum preparation, cultures were grown on fresh potato dextrose agar (PDA; $250 \mathrm{~g}$ of unpeeled potato, $20 \mathrm{~g}$ of agar, and $20 \mathrm{~g}$ of glucose per liter of distilled water) for 7 to 10 days at $24^{\circ} \mathrm{C}$ in the dark.

Generation and characterization of nit mutants. nit mutants were generated according to Korolev and Katan (36) on water agar-chlorate (WAC) medium consisting of $2 \%$ agar, $0.02 \%$ glucose, and $5 \%$ potassium chlorate. Small mycelial plugs from 7- to

TABLE 1. Isolates of Verticillium dahliae from the Comunidad Valenciana region in eastern-central Spain used in this study, listed by vegetative compatibility group (VCG), reference number, source, and molecular marker

\begin{tabular}{|c|c|c|c|c|}
\hline \multirow[b]{2}{*}{ VCG } & \multirow[b]{2}{*}{ Isolate } & \multicolumn{2}{|c|}{ Source } & \multirow[b]{2}{*}{ Molecular marker $(\mathrm{bp})^{\mathrm{a}}$} \\
\hline & & Host & Province & \\
\hline \multirow[t]{2}{*}{ VCG1A } & V610, V611, V612 & Cotton & Alicante & 334 and 462 \\
\hline & V403 & Artichoke & Valencia & 334 and 462 \\
\hline \multirow[t]{4}{*}{ VCG2A } & V485, V541, V542, V548, V573, V575-V579, V582-V586, V588, V589, V591, & & & \\
\hline & V592, V595, V689-V692, V694, V696 & Artichoke & Alicante & 824 \\
\hline & V927 & Artichoke & Castellón & 824 \\
\hline & V404, V707, V917, V918 & Artichoke & Valencia & 824 \\
\hline \multirow[t]{3}{*}{ VCG2B } & $\begin{array}{l}\text { V538-V540, V545, V546, V570, V574, V587, V590, V593, V594, V693, V695, } \\
\text { V697-V700, V921-V926 }\end{array}$ & Artichoke & Alicante & 334 or 824 \\
\hline & V396, V400, V474, V475, V479-V484, V530-V536, V571, V572, V613,V928 & Artichoke & Castellón & 334 or 824 \\
\hline & $\begin{array}{l}\text { V402, V407, V473, V476, V569, V477, V549, V550, V675-V682, V685, V686, } \\
\text { V688, V701-V706, V708, V919, V920 }\end{array}$ & Artichoke & Valencia & 334 or 824 \\
\hline VCG4B & V683, V684, V687 & Artichoke & Valencia & 824 \\
\hline $\mathrm{HSI}^{\mathrm{b}}$ & V537, V547 & Artichoke & Alicante & 334 \\
\hline
\end{tabular}

a Amplicons produced in polymerase chain reaction assays using primer sets DB19/DB22/espdef01 (334 bp), INTD2f/INTD2r (462 bp), and INTND2f/INTND2r (824 bp). Amplicons of 334 and 462 bp previously were associated with the cotton-defoliating $V$. dahliae pathotype, and that of 824 bp previously was associated with the cotton-nondefoliating pathotype (41-43).

${ }^{\mathrm{b}} \mathrm{HSI}=$ heterokaryon self-incompatible. 
10-day-old $V$. dahliae cultures were placed at six to eight points in petri plates $\left(9 \mathrm{~cm}\right.$ in diameter) and incubated at $24^{\circ} \mathrm{C}$ for 2 to 3 weeks in the dark. Chlorate-resistant sectors, evident as fastgrowing sectors after 10 to 15 days, were transferred to CDA plates and further incubated under the same conditions. Sectors that grew on CDA as thin, expansive colonies with no aerial mycelium were considered nit mutants. nit mutants were partially phenotyped on CDA amended with sodium nitrite $(0.5 \mathrm{~g} / \mathrm{liter})$ or hypoxanthine ( $0.2 \mathrm{~g} / \mathrm{liter})$ as described by Correll and Leslie (20).

Vegetative compatibility grouping. Complementation tests were done by pairing phenotypically distinct nit mutants of an isolate, as well as by pairing them with nit mutants of the international OARDC (The Ohio State University, Woodster, $\mathrm{OH}$ ) reference strains of $V$. dahliae VCGs (VCG1, strain T9M; VCG3, strain 70-21; and VCG4A, strains 131M and 171) and Israeli nit testers (VCG1, strain Cot200; VCG2A, strains Ep8M and Ep52; VCG2B, strains Cot11M and Cot254; and VCG4B, strains Pt15M and $\mathrm{Pt9G}$ ). All reference testers were kindly provided by T. Katan (ARO, The Volcani Center, Bet Dagan, Israel). Israeli nit testers were proved to correlate with the international OARDC reference strains $(34,37)$. According to Bell (7), the D strain T9 belongs to subgroup VCG1A and only isolates of this subgroup caused

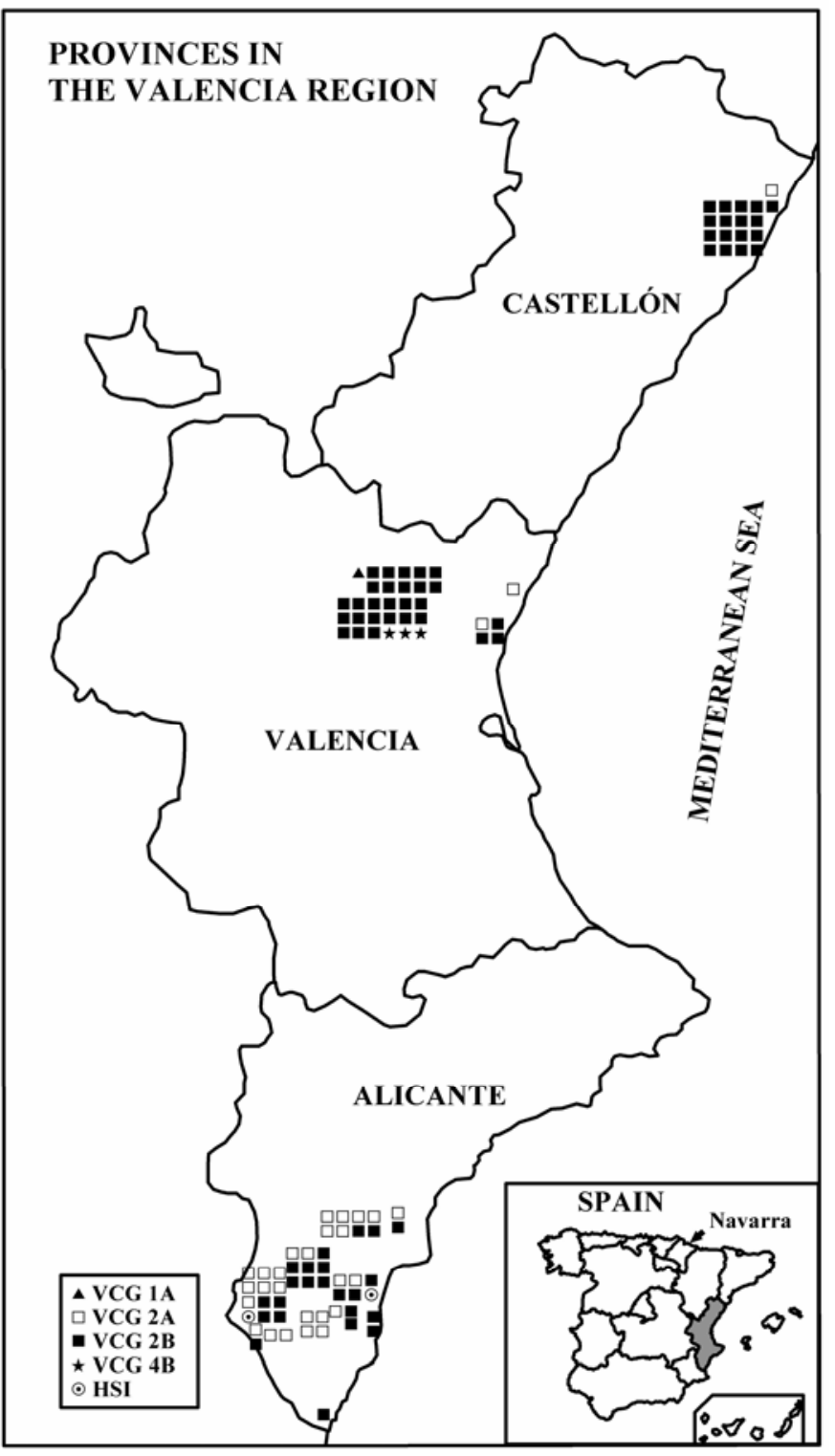

Fig. 1. Regional distribution of vegetative compatibility groups (VCGs) of Verticillium dahliae from artichoke in the Comunidad Valenciana region in eastern-central Spain. severe defoliation of cotton in previous studies $(21,39,58)$. Thus, isolates strongly compatible with $\mathrm{T} 9$ (or with other testers strongly compatible with it) may be assigned to VCG1A (39). In order to distinguish between putative subgroups of VCG1 (VCG1A and VCG1B) (7), nit mutants were generated from $V$. dahliae D isolate V138, assigned to VCG1 by Korolev et al. (39), and isolates 1990.1 and 9.6 from Japanese maple (Acer palmatum Thunb.) and yellow wood (Cladastris lutea (F. Michx.) K. Koch), respectively (15). Isolates 1990.1 and 9.6 presumably are representatives of VCG1B (7). Pairing was done by placing mycelial plugs of mutants 1 to $1.5 \mathrm{~cm}$ apart on CDA in petri plates $(9 \mathrm{~cm}$ in diameter) at $24^{\circ} \mathrm{C}$ in the dark; nit mutants of test and tester isolates were paired in all possible combinations. Plates were scored for prototrophic growth after 14 and 28 days of incubation. Complementation was indicated by the formation of a dense, aerial growth where mycelia from an unknown and tester strain had met and formed a prototrophic heterokaryon. In such a case, the unknown mutant was considered vegetatively compatible with the tester strain and was assigned to the VCG of the tester strain.

Specific PCR assays. All $112 \mathrm{~V}$. dahliae isolates in this study were molecularly characterized by means of PCR assays using several primer pairs. Use of primers DB19 and DB22 (12) produces amplicons of 539 or 523 bp which are specific to V. dahliae, but are differentially associated with $\mathrm{D}$ and ND $V$. dahliae isolates, respectively (41). Those two amplicons differ in a 15-nucleotide (nt) insertion that was the basis for designing a new primer, espdef01 (41). The use of primers DB19, DB22, and espdef01 in a single reaction yields one of the 523- or 539-bp markers together with an amplicon of $334 \mathrm{bp}$ in the case of D $V$. dahliae isolates $(19,41)$. Similarly, the use of primer pairs INTND2f/INTND2 $r$ and INTD2f/INTD2 $r$ produces either an amplicon of $824 \mathrm{bp}$ from ND isolates or of $462 \mathrm{bp}$ from D isolates $(42,43)$; and that of primer pairs INTND2f/INTND2 $r$ and DB19/espdef01 yields the 824- or 334-bp amplicons from ND and D V. dahliae isolates, respectively (41).

Mycelia of isolates grown in supplemented Czapek-Dox broth were harvested, lyophilized, and ground as described previously (46). DNA extracted from ground mycelia by the method of Möller et al. (44) was used for PCR assays. Amplifications using primer sets DB19/DB22 and DB19/DB22/espdef01were carried out according to Mercado-Blanco et al. (41) with minor modifications. Briefly, the reaction mixture $(25 \mu \mathrm{l})$ consisted of a $\mu \mathrm{M}$ ratio of 0.7:0.1:0.2 (DB19/DB22/espdef01) for primers, $200 \mu \mathrm{M}$ each $\mathrm{dNTP}, 2.5 \mu \mathrm{l}$ of $10 \times$ reaction buffer, $0.75 \mathrm{U}$ of EcoTaq, $1.5 \mathrm{mM}$ $\mathrm{MgCl}_{2}$, and $10 \mathrm{ng}$ of template DNA. Reaction conditions included denaturation at $94^{\circ} \mathrm{C}$ for $4 \mathrm{~min}$ followed by 30 cycles of $1 \mathrm{~min}$ of annealing at $60^{\circ} \mathrm{C}$, extension for $30 \mathrm{~s}$ at $72^{\circ} \mathrm{C}$, denaturation for $1 \mathrm{~min}$ at $94^{\circ} \mathrm{C}$, and a final extension step of $6 \mathrm{~min}$ at $72^{\circ} \mathrm{C}$. Amplifications using DB19/DB22 primers were carried out at the same conditions as above, except that primers were added at $0.4 \mu \mathrm{M}$ each, the annealing temperature was set at $54^{\circ} \mathrm{C}$, and 30 cycles were run. Amplifications using primer pairs INTND2f/INTND2r (824 bp) and INTD2f/INTD2r (462 bp) or INTND2f/INTND2r and DB19/espdef01 (334 bp) were done at the same conditions as for primers DB19/DB22/espdef01, except that $0.2 \mu \mathrm{M}$ each primer was used and 30 cycles were run with the annealing temperature set at $64^{\circ} \mathrm{C}$ for $1 \mathrm{~min}$ for the former set of primer pairs, and 30 cycles at $60^{\circ} \mathrm{C}$ were run for the second one. The final extension step was at $72^{\circ} \mathrm{C}$ for 6 and $10 \mathrm{~min}$, respectively (41-43).

All PCR assays were performed with a PTC-100 Programmable Thermal Controller (MJ Research, Inc., Watertown, MA). Amplification products were separated on $1 \%$ agarose gels stained with ethidium bromide and visualized under UV light. The DNA molecular weight marker XIV (100-bp ladder) used for electrophoresis was from Roche Diagnostics (Mannheim, Germany). All primers used in this study were obtained from Genset Oligos (Genset SA, Paris, France). Reactions were repeated at least twice and always included negative controls (no DNA) and 
positive controls (template DNA from $V$. dahliae isolates representative of D and ND pathotypes from previous studies) (41-43).

Pathogenicity tests. Two experiments (I and II) were carried out using 35 and 39 selected, monoconidial $V$. dahliae isolates, respectively. These isolates were chosen to represent their geographic range in the Comunidad Valenciana region as well as the VCG and molecular diversity found in this study (Table 1). Thirty-three isolates were common in the two experiments. In experiment I, 33 isolates from artichoke and 2 isolates from cotton were compared for virulence to artichoke by the root-dip inoculation method; these 35 isolates were from VCG1A ( 3 isolates), VCG2A (8 isolates), VCG2B (21 isolates), and VCG4B (1 isolates), and 1 isolate was heterokaryon self-incompatible (HSI). In experiment II, 37 isolates from artichoke and 2 cotton isolates were compared for virulence to cotton by the stem-injection method; these isolates belonged to VCG1A (3 isolates), VCG2A (8 isolates), VCG2B (22 isolates), and VCG4B ( 2 isolates), and 4 isolates were HSI. In addition, isolates V117I (VCG1A) and V176I (VCG2A), representative of the D and ND pathotypes (35), respectively, were included in experiment II for comparison.

Experiment I was performed at Instituto Agroforestal Mediterráneo, Universidad Politécnica, Valencia. For root-dip inoculation (39), disinfested ( $1 \% \mathrm{NaClO}, 2 \mathrm{~min})$, germinated seed of artichoke hybrid cvs. Nun 6374 and Nun 9444 (Nunhems Seeds Ltd., Valencia, Spain) were sown in trays filled with a twice-autoclaved $\left(120^{\circ} \mathrm{C}\right)$ potting mixture (peat/sand, $2: 1$, vol/vol). Plants were grown in a greenhouse at 20 to $24^{\circ} \mathrm{C}$ for 6 weeks (three to four true leaves fully formed) until inoculation. Inoculum was obtained by flooding 7- to 10-day-old cultures on PDA with sterile distilled water (SDW) and filtering the suspension through sterile cheesecloth. The conidial density in the suspension was adjusted to $10^{6}$ conidia $\mathrm{ml}^{-1}$ for each of the isolates. Plants were uprooted from the trays and their roots were washed thoroughly in tap water to remove substrate residues, slightly trimmed, and dipped for $10 \mathrm{~min}$ in the conidial suspension of an isolate. Noninoculated control plants were dipped in SDW. Plants then were transplanted (one per pot) to $13-\mathrm{cm}$-diameter plastic pots filled with the autoclaved potting mixture and incubated in the greenhouse at the same conditions described above for 2 months.

Experiment II was carried out at CIFA-IFAPA, Córdoba. For stem-injection inoculation (8), disinfested (1\% $\mathrm{NaClO}, 2 \mathrm{~min})$, germinated seed of cotton cv. Acala SJ-2 were sown in 12-cm-diameter plastic pots (one plant per pot) filled with an autoclaved potting mixture (sand/clay loam/peat, 2:1:2, vol/vol/vol). Plants were grown in a greenhouse at 18 to $24^{\circ} \mathrm{C}$. Six-week-old plants were inoculated with $5 \mu \mathrm{l}$ of a $3 \times 10^{6}$ conidia $\mathrm{ml}^{-1}$ suspension in SDW at each of two opposite points at the base of the first and second stem internodes. Control plants were treated similarly with SDW. After inoculation, plants were maintained in the greenhouse as before.

Plants were watered as needed and fertilized with a watersoluble fertilizer (20-10-20, N-P-K) every 2 weeks. Plants were observed periodically for the development of foliar symptoms. Severity of symptoms in individual plants was assessed according to percentage of foliar tissue affected in acropetal progression. A 0 -to-5 rating scale $(0=$ no symptoms; $1=1$ to $25,2=26$ to 50 , $3=51$ to 75 , and $4=76$ to $100 \%$ diseased; and $5=$ dead plant) was used at 1-week intervals for 8 weeks after inoculation for experiment I, and a 0 -to- 4 rating scale $(0=$ no symptoms; $1=1$ to $33,2=34$ to 66 , and $3=67$ to $100 \%$ diseased; and $4=$ dead plant) at 2, 3, and 4 weeks after inoculation $(8,39)$ for experiment II. Because different rating scales and procedures were used in the two experiments, a disease intensity index (DII) was calculated to facilitate visualizing the trend of disease reactions in artichoke and cotton according to the equation $D I I_{i}=S_{i} 100 / S_{m x}$, where $S_{i}=$ symptom severity at $i$ assessment and $S_{m x}=$ maximum symptom severity (i.e., $S_{m x}=5$ in experiment I and 4 in experiment II). Thus, DII expresses the severity of symptoms in a plant at any given moment as a percentage of the maximum possible severity value. Disease progress curves were obtained by plotting DII values over time. The area under the disease progress curve (AUDPC) calculated by the trapezoidal integration method (11) was expressed as percentage of the maximum possible area for the duration of experiments. At the termination of experiment II, cotton plants were excised above the cotyledon node for fresh weight determination. Also, at the end of experiments, isolations were carried out from symptomatic and nonsymptomatic plants for confirmation of infection by the pathogen. Pieces from artichoke petioles and cotton stems were surface disinfested in $0.5 \%$ $\mathrm{NaClO}$ for $2 \mathrm{~min}$, cut into 5- to 10-mm-long fragments, plated on PDA, and incubated at $24^{\circ} \mathrm{C}$ in the dark for 7 days.

Experiments I and II had two-factor ( $V$. dahliae isolates $\times$ artichoke cultivars) and one-factor ( $V$. dahliae isolates $\times$ a single cotton cultivar) treatment designs, respectively. Treatments were replicated according to a randomized complete block design with five and nine replicates (each replicate consisting of a single potted plant) for experiments I and II, respectively. Data on the final DII (i.e., determined 8 weeks after inoculation in experiment I and 4 weeks after inoculation in experiment II), AUDPC, and fresh weight were subjected to analysis of variance using the general linear model procedure of SAS (version 6.11; SAS Institute, Cary, NC). No statistical comparisons were made between results in the two experiments because of differences in the rating scales and experimental procedures between them. Percentage data were transformed into arcsine $(Y / 100)^{1 / 2}$ before analysis. Experiments were repeated once. Similarity among repetitions of an experiment tested by preliminary analysis of variance using experimental runs as blocks allowed combining data for analyses. Linear single-degree-of-freedom contrasts were computed to test the effect of selected experimental treatment combinations (29).

\section{RESULTS}

Vegetative compatibility of $\boldsymbol{V}$. dahliae isolates. All 109 $V$. dahliae isolates from artichoke and $3 \mathrm{~V}$. dahliae isolates from cotton in the study produced chlorate-resistant sectors on WAC. Of those sectors, 60 to $100 \%$ were considered nit mutants in $87.8 \%$ of isolates, because they formed expansive colonies with thin mycelium on CDA. A total of $\approx 1,500$ nit mutants were recovered and characterized to phenotype for the $224 \mathrm{~V}$. dahliae cultures (two monoconidial cultures of each of 112 isolates) in the study. Of those nit mutants, $87.8 \%$ were classified as nitl and $12.2 \%$ as NitM. No nit3 mutants were identified. At least one nit mutant was recovered from each of the $112 \mathrm{~V}$. dahliae isolates, but no NitM was recovered from 26 isolates. Therefore, 86 isolates could be tested for self-compatibility, of which 2 from artichoke in Alicante were HSI.

In 89 of 110 isolates, complementing heterokaryons formed between different mutant phenotypes (nitl and NitM) of the tested isolates and the international OARDC reference strains and Israeli tester strains. The timing and morphology of heterokaryons in pairings of compatible nit mutants varied from fast to slow prototrophic growth and formation of dense to scattered aerial mycelium with or without development of microsclerotia. Based on their positive complementation reactions with specific testers, the 89 isolates were assigned to four VCGs: VCG1, VCG2 (comprising VCG2A and VCG2B), and VCG4B, with the two monoconidial cultures of each isolate being assigned to same VCG. nit mutants of VCG1 cotton isolate V138 and VCG1B isolates 1990.1 and 9.6 were paired in all possible combinations with complementing nit mutants of isolates V403 (artichoke) and V610, V611, and V612 (cotton) (Table 1) as well as of the T9M and Cot200 VCG1A reference strains from cotton. Heterokaryon formation between complementing mutants of cotton and the artichoke isolates was strong to moderate, depending on isolate, but generally stronger than pairings between them and mutants from isolates 
1990.1 and 9.6. Thus, based on the vigor of heterokaryons, the three isolates from cotton and the one artichoke isolate were assigned to VCG1A. Of the 85 remaining isolates from artichoke, 31 were assigned to VCG2A, 51 to VCG2B, and 3 to VCG4B.

nit mutants of 21 of the tested isolates failed to establish compatible heterokaryons when paired with complementing mutants (nitl and NitM) of the international reference and Israeli tester strains in all possible combinations. No NitM was recovered from 9 of those 21 isolates. nitl mutants of the 21 isolates were further paired in all possible combinations with NitM mutants of selected, tested isolates from artichoke that previously had been assigned to a VCG based on their compatibility with the international reference and Israeli tester strains. This allowed all 21 isolates to be assigned to VCG2B. nitl mutants of those 21 isolates were further proved compatible with NitM mutants of VCG2B artichoke isolates V396 (824-bp molecular pattern) as well as V549 (334-bp molecular pattern) (see below). Moreover, nitl mutants of the 9 isolates for which no NitM was recovered formed strong heterokaryons with NitM mutants of 5 isolates arbitrarily chosen among 12 self-compatible isolates of the 21 VCG2B isolates for which self-compatibility could be tested. Two subgroups, VCG2Br and VCG2Ba, were differentiated among $V$. dahliae isolates from artichoke belonging to $\mathrm{VCG} 2 \mathrm{~B}$; the former, VCG2Br, included isolates compatible with the international reference and Israeli tester strains, and the latter, VCG2Ba, included isolates compatible with NitM mutants from VCG2Br isolates collected from Spain but not with the reference tester strains. Thus, in all, $107 \mathrm{~V}$. dahliae isolates from artichoke were assigned to VCGs: 1 isolate $(0.9 \%)$ to VCG1A, $31(28.9 \%)$ to VCG2A, 72 $(67.3 \%)$ to VCG2B (47.7\% VCG2Br and $19.6 \%$ VCG2Ba), and 3 $(2.8 \%)$ to VCG4B, and 2 isolates were HSI (Table 2).

Prevalence of the identified VCGs varied among provinces in the surveyed region (Table 2). All but one isolate from Castellón province in the northern part of the region (Fig. 1) were of VCG2B, of which $95.2 \%$ were of VCG2Br. Consequently, the two isolates sampled from each of eight fields in that province were of VCG2B. Conversely, isolates from Valencia, in the center of the region, showed the highest VCG diversity; $77.8 \%$ of isolates were assigned to the predominant group VCG2B $(50.0 \%$ VCG2Br and $27.8 \% \mathrm{VCG} 2 \mathrm{Ba}$ ), four isolates (11.1\%) belonged to VCG2A, three $(8.3 \%)$ to VCG4B, and one $(2.8 \%)$ to VCG1A. For the 13 fields in the Valencia province from which two isolates were collected, the two isolates were of VCG2B in 10 fields and VCG4B in one field but they belonged to different VCGs in the two fields remaining (VCG2A and VCG2B in one field, VCG2B and VCG4B in another field). Isolates from Alicante, in the southern part of the region, were almost equally distributed between VCG2A $(53.1 \%)$ and VCG2B (46.9\% total: $26.5 \%$ VCG2Br and $20.4 \%$ VCG2Ba). In 10 of 17 fields in this province from which two isolates were collected, they were of the same VCG, VCG2A (7 fields) and VCG2B (3 fields); however, for 6 of those 17 fields, the isolates belonged to either VCG2A or VCG2B and, in 1 field, the isolates were of VCG2B or HSI. Therefore, VCG2B was widespread in the surveyed area, whereas VCG1A and VCG4B were found in Valencia only, and $83.9 \%$ of all VCG2A isolates were from Alicante.

PCR analysis. When genomic DNA from all four $V$. dahliae isolates from artichoke and cotton assigned to VCG1A were amplified in single- or duplex-PCR assays using primers DB19/ DB22/espdef01 or INTND2f/INTND2r (824 bp) and INTD2f/ INTD2r (426 bp), respectively, only the 334- and 462-bp amplicons diagnostic of the D pathotype were obtained (Table 1). Similarly, for isolates assigned to VCG2A and VCG4B, duplex-PCR assays using primer pairs INTND2f/INTND2r and INTD2f/ INTD2r or INTND2f/INTND2r and DB19/espdef01 (334 bp) yielded only the 824-bp amplicon diagnostic of ND $V$. dahliae

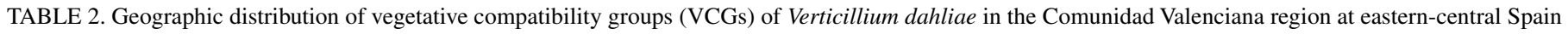

\begin{tabular}{|c|c|c|c|c|c|c|c|c|}
\hline \multirow[b]{3}{*}{ Province } & \multirow[b]{3}{*}{ Host } & \multicolumn{7}{|c|}{ Number of isolates } \\
\hline & & \multirow[b]{2}{*}{ VCG1A } & \multirow[b]{2}{*}{$\mathrm{VCG} 2 \mathrm{~A}$} & \multicolumn{2}{|c|}{$\mathrm{VCG}_{2} \mathrm{~B}^{\mathrm{a}}$} & \multirow[b]{2}{*}{ VCG4B } & \multirow[b]{2}{*}{$\mathrm{HSI}^{\mathrm{b}}$} & \multirow[b]{2}{*}{ Total } \\
\hline & & & & VCG2Br & VCG2Ba & & & \\
\hline \multirow{2}{*}{ Alicante } & Cotton & 3 & 0 & 0 & 0 & 0 & 0 & 3 \\
\hline & Artichoke & 0 & 26 & 13 & 10 & 0 & 2 & 51 \\
\hline Castellón & Artichoke & 0 & 1 & 20 & 1 & 0 & 0 & 22 \\
\hline Valencia & Artichoke & 1 & 4 & 18 & 10 & 3 & 0 & 36 \\
\hline Total & $\ldots$ & 4 & 31 & 51 & 21 & 3 & 2 & 112 \\
\hline
\end{tabular}

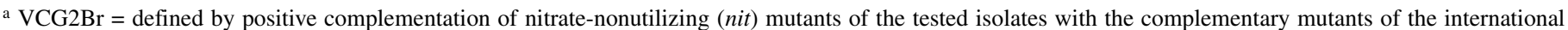
OARDC reference and Israeli tester strains $(37,49)$. VCG2Ba = defined by positive complementation of nit 1 mutants of the tested isolates with selected NitM mutants of isolates from artichoke that previously had been assigned to VCG2Br. Isolates assigned to VCG2Ba failed to establish positive complementation with nit mutants of the international reference and Israeli tester strains.

${ }^{\mathrm{b}} \mathrm{HSI}=$ heterokaryon self-incompatible.

TABLE 3. Molecular diversity in Verticillium dahliae isolates from artichoke in the Comunidad Valenciana region in eastern-central Spain belonging to vegetative compatibility group (VCG) VCG2B

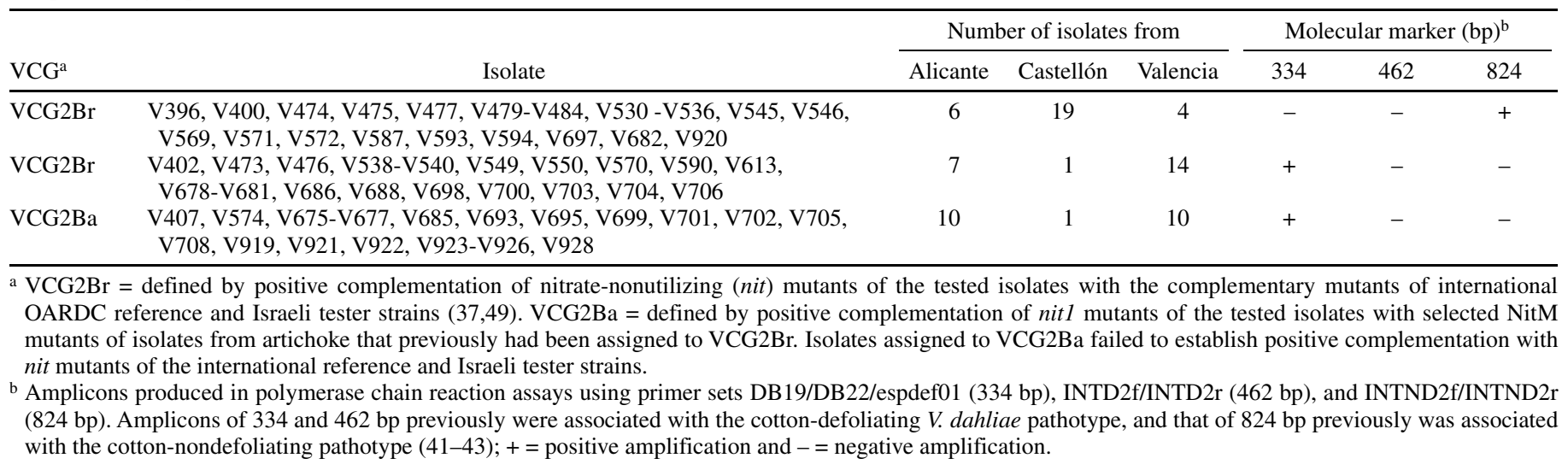


(Table 1). Conversely, results of similar PCR assays using genomic DNA from isolates belonging to VCG2Br and VCG2Ba indicated molecular diversity within VCG2B (Table 3). Thus, all 21 VCG2Ba isolates from the three sampled provinces yielded the 334-bp amplicon previously associated with the D pathotype in PCR assays using primers DB22, DB19, and espdef01, but neither the 462- nor the 824-bp markers previously associated with the D and ND pathotypes, respectively, in amplifications using primer pairs INTD2f/INTD2r and INTND2f/INTND2r. Similarly, $43.1 \%$ of the 51 isolates assigned to $\mathrm{VCG} 2 \mathrm{Br}$ in the three provinces produced the same 334-bp pattern of amplification as those in VCG2Ba, but the remaining $56.9 \%$ of them yielded the 824-bp pattern of amplification shown by isolates in VCG2A and VCG4B (Table 3). Although most VCG2Br isolates from Castellón were of the 824-bp pattern, the 334-bp pattern predominated among VCG2Br isolates from Valencia, and the two molecular patterns were distributed equally among VCG2Br isolates from Alicante. The two HSI isolates identified showed the 334-bp pattern.

Virulence of $V$. dahliae isolates to artichoke and cotton cultivars. All $35 \mathrm{~V}$. dahliae isolates from artichoke (33 isolates) and cotton ( 2 isolates) tested in experiment I were pathogenic to artichoke. Symptoms developed by 10 days after inoculation and consisted of chlorosis, wilting, and necrosis of basal leaves which progressed upward on the plant to reach the newly formed ones. There was no defoliation in affected plants. Some of the inoculated plants died by 60 days after inoculation, the number of dead plants varying with the virulence of the isolates. No symptoms developed in uninoculated controls.

Analysis of variance of the transformed data on disease reaction indicated significant $(P<0.05)$ differences in virulence among $V$. dahliae isolates as well as in susceptibility between artichoke cultivars, with cv. Nun 6374 being significantly $(P<0.05)$ more susceptible than cv. Nun 9444. Mean final DII and AUDPC were 89.5 and 44.9, respectively, for cv. Nun 6734 and 84.0 and 42.0 for cv. Nun 9444 . However, the isolate-cultivar interaction was not statistically significant. Therefore, combined data for the two cultivars were used to compute linear single-degree-offreedom contrasts for comparison between virulence of isolates according to VCG. Further statistical analyses using nontransformed disease severity (DS) data confirmed the significance of differences in virulence between isolates according to VCG as well as in susceptibility between artichoke cultivars. Collectively, isolates in VCG2B (including $\mathrm{VCG} 2 \mathrm{Br}$ and $\mathrm{VCG} 2 \mathrm{Ba}$ ) were significantly $(P<0.001)$ more virulent to artichoke than those in VCG2A, as indicated by the final DS, final DII, and AUDPC (Table 4). However, both VCG2A and VCG2B collectively

TABLE 4. Virulence of Verticillium dahliae isolates from artichoke and cotton in the Comunidad Valenciana region in eastern-central Spain to artichoke cvs. Nun 6374 and $9444^{\mathrm{a}}$

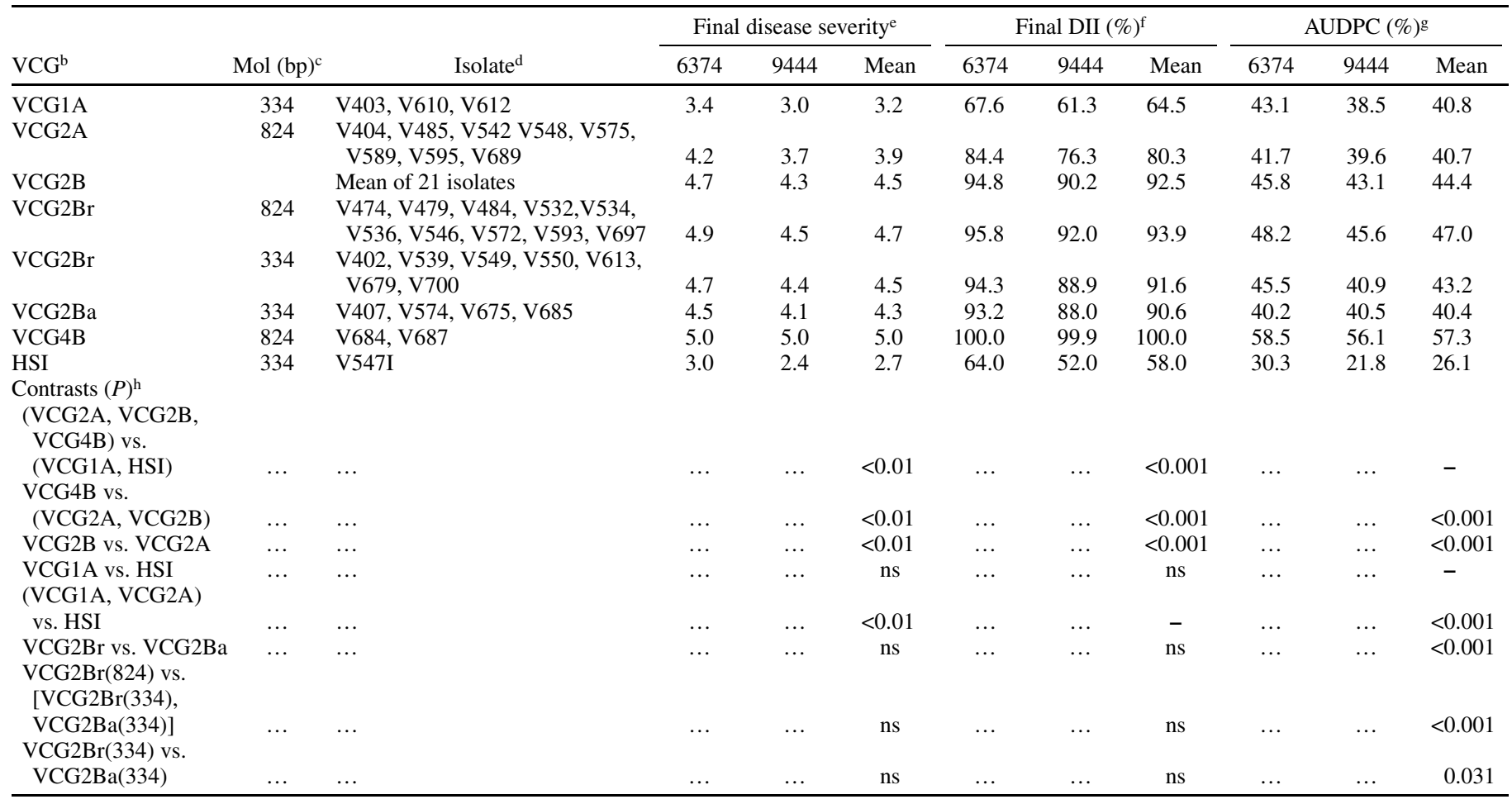

a Data are means of 10 replicated plants per treatment combination. Plants were inoculated by dipping their bare, trimmed roots in a $10^{6}$ conidia/ml suspension of an isolate for $10 \mathrm{~min}$. VCG = vegetative compatibility group.

${ }^{\mathrm{b}} \mathrm{VCG} 2 \mathrm{Br}=$ defined by positive complementation of nitrate-nonutilizing (nit) mutants of the tested isolates with the complementary mutants of international OARDC reference and Israeli tester strains $(37,49)$. VCG2Ba = defined by positive complementation of nitl mutants of the tested isolates with selected NitM mutants of isolates from artichoke that previously had been assigned to VCG2Br. Isolates assigned to VCG2Ba failed to establish positive complementation with nit mutants of the international reference and Israeli tester strains. HSI = heterokaryon self-incompatible.

${ }^{\mathrm{c}}$ Molecular markers. Amplicons produced in polymerase chain reaction assays using primer sets DB19/DB22/espdef01 (334 bp) and INTND2f/INTND2r (824 bp). Amplicons of 334 and 824 bp previously were associated with the cotton-defoliating and -nondefoliating pathotypes of $V$. dahliae, respectively (41-43).

d All isolates were monoconidial cultures from Verticillium wilt-affected artichoke in the region, except for isolates V610 and V612 that infected cotton in it.

${ }^{\mathrm{e}}$ Mean disease severity assessed on a 0 -to- 5 rating scale (where $0=$ no symptoms and $5=$ dead plants) 8 weeks after inoculation.

${ }^{\mathrm{f}}$ Disease intensity index (DII) determined 8 weeks after inoculation by the equation $D I I_{\mathrm{i}}=S_{\mathrm{i}} 100 / 5$, where $S_{\mathrm{i}}=$ symptom severity at $i$ assessment on a scale of 0 (no symptoms) to 5 (dead plant).

$\mathrm{g}$ Area under the DII progress curve (AUDPC) over time calculated by the trapezoidal integration method (11) and expressed as a percentage of the maximum possible area for the duration of the experiment.

${ }^{\mathrm{h}}$ Linear single-degree-of-freedom contrast computed to test the effect of selected treatment combinations. Probability for the $t$ statistic of linear single-degree-offreedom contrasts; - indicates the comparison was not performed and $\mathrm{ns}=$ not significant $(P>0.05)$. 
appeared to be significantly $(P<0.001)$ less virulent than VCG4B (Table 4). Conversely, isolates in VCG1A were as virulent as those being HSI and both collectively showed the least virulence to artichoke compared with the other VCGs, as indicated by comparisons of the final DS and DII (Table 4). HSI isolates and isolates of VCG1A and VCG2A, in this order, appeared the least virulent to artichoke. Some differences in virulence also occurred among isolates in VCG2B that were associated with genetic and molecular diversity within this VCG. Thus, isolates in VCG2Br were significantly $(P<0.001)$ more virulent than those in VCG2Ba, as indicated by the AUDPC but not by the final DS and DII; and the same occurred for isolates in the VCG2Br subgroup of VCG2B that amplified the 824-bp amplicon compared with isolates in VCG2B that amplified the 334-bp amplicon (i.e., those in VCG2Ba and the second molecular subgroup of VCG2Br) (Table 4).

All $39 \mathrm{~V}$. dahliae isolates from artichoke (37 isolates) and cotton ( 2 isolates) tested in experiment II were pathogenic to cotton cv. Acala SJ-2, of which 33 isolates were common to experiment I, including the 3 VCG1A isolates from artichoke (V403) and cotton (V610 and V612). However, there were statistically significant $(P<0.001)$ differences in virulence among isolates according to VCG, and these differences were confirmed by further statistical analyses using nontransformed disease severity data (Table 5). No symptoms developed in control plants, which had a mean fresh weight of $13.5 \mathrm{~g} / \mathrm{plant}$ (data not shown). Symptoms in inoculated plants developed by 2 weeks after inoculation. All three tested VCG1A isolates from artichoke or cotton, as well as the VCG1A isolate V117I from cotton included for comparison, caused defoliation and stunting of the plant (mean fresh weight $4.0 \mathrm{~g} / \mathrm{plant}$ ) (Table 5) characteristic of the D pathotype (8,39). Isolates belonging to other VCGs induced mild to severe foliar symptoms but caused neither defoliation nor stunting (mean fresh weight ranged from 8.0 to $9.5 \mathrm{~g} /$ plant) (Table 5).

Linear single-degree-of-freedom contrasts indicated that the virulence pattern of isolates of a VCG to cotton (Table 5) varied when compared with the virulence pattern shown by isolates of the same VCG to artichoke (Table 4). Thus, isolates of VCG1A were significantly $(P<0.001)$ more virulent to cv. Acala SJ-2 than isolates of VCG2A, VCG2B, VCG4B, and HSI isolates, collectively, and such a difference was indicated by the final DS, final DII, AUDPC, and plant fresh weight (Table 5). Compared with disease reactions on artichoke, isolates of VCG2B (including $\mathrm{VCG} 2 \mathrm{Br}$ and VCG2Ba) were not significantly different in virulence to cotton compared with those of VCG2A, VCG4B, and HSI isolates, collectively (Tables 4 and 5). However, as shown for virulence to artichoke, isolates of subgroup $\mathrm{VCG} 2 \mathrm{Br}$ were significantly $(P<0.01)$ more virulent to cotton than isolates of VCG2Ba, and that was indicated by differences in the final DS, final DII, and AUDPC, but not in the plant fresh weight (Table 5). Similarly, VCG2Br isolates that amplified the 824-bp amplicon were significantly $(P<0.001)$ more virulent than isolates of VCG2B that amplified the 334-bp amplicon (i.e., including isolates of VCG2Ba and isolates of the other molecular subgroup of $\mathrm{VCG} 2 \mathrm{Br}$ ).

TABLE 5. Virulence of Verticillium dahliae isolates from artichoke and cotton in the Comunidad Valenciana region in eastern-central Spain to cotton cv. Acala SJ-2a

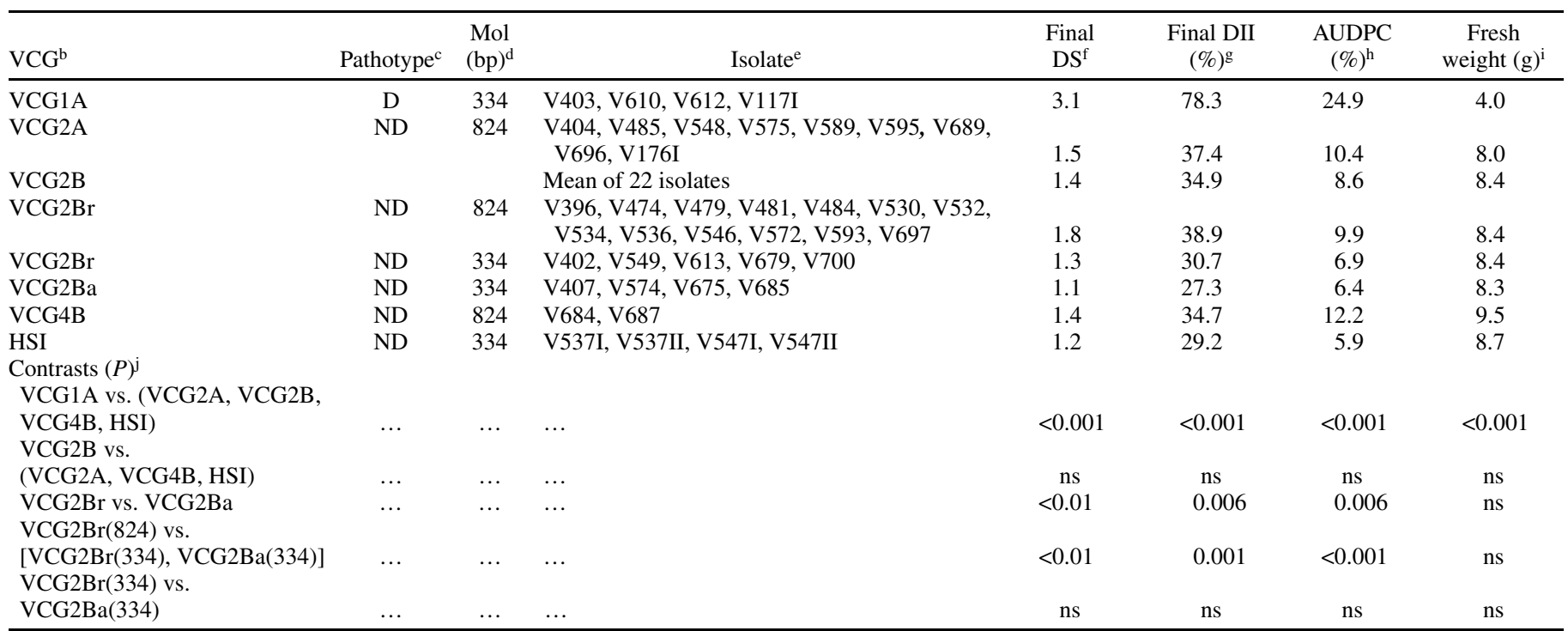

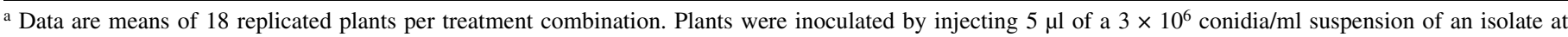
each of two opposite points at the base of the first and second stem internodes.

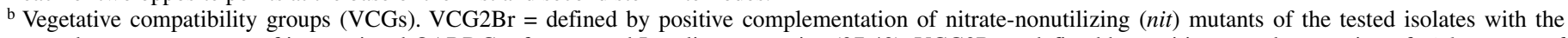
complementary mutants of international OARDC reference and Israeli tester strains $(37,49)$. VCG2Ba $=$ defined by positive complementation of nit 1 mutants of the tested isolates with selected NitM mutants of isolates from artichoke that previously had been assigned to VCG2Br. Isolates assigned to VCG2Ba failed to establish positive complementation with nit mutants of the international reference and Israeli tester strains. HSI $=$ heterokaryon self-incompatible.

${ }^{\mathrm{c}} \mathrm{D}=$ cotton-defoliating pathotype and $\mathrm{ND}=$ cotton-nondefoliating pathotype.

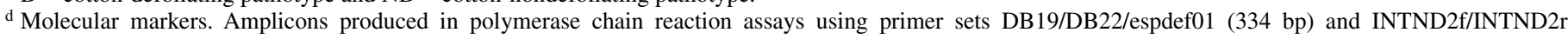
(824 bp). Amplicons of 334 and 824 bp previously were associated with the D and ND pathotypes of $V$. dahliae, respectively (41-43).

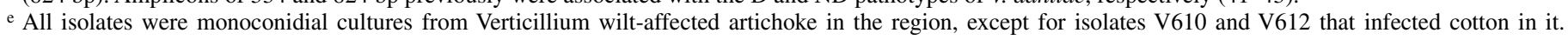
Isolates V117I and V176I are reference cotton isolates of the D and ND pathotypes, respectively (8,39). Isolates 537I and 537II, and isolates 547I and 547II, are the two monoconidial cultures of each of parental isolates used for VCG characterization.

${ }^{\mathrm{f}}$ Mean disease severity (DS) assessed on a 0-to-4 rating scale (where $0=$ no symptoms and $4=$ dead plant) 4 weeks after inoculation.

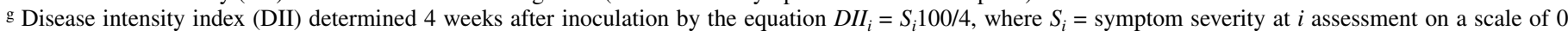
(no symptoms) to 4 (dead plant).

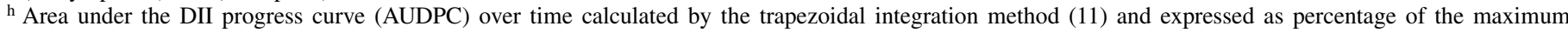
possible area for the duration of the experiment.

i Determined on stems excised above the cotyledon node 4 weeks after inoculation.

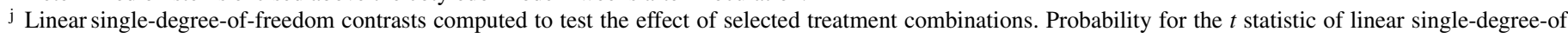
freedom-contrasts; $\mathrm{ns}=$ not significant $(P>0.05)$. 


\section{DISCUSSION}

Phenotypic and genetic characterization of prevailing $V$. dahliae populations is of importance for the effective management of Verticillium wilt in artichoke-growing areas. In this study, examination of a large sample of artichoke $V$. dahliae isolates for vegetative compatibility, molecular markers, and virulence to artichoke and cotton indicated that VCG2 prevails among the four VCGs (VCG1A, VCG2A, VCG2B, and VCG4B) identified in the most important artichoke production area of Spain, with VCG2B being the predominant subgroup. More importantly, we demonstrated that there was some correlation with VCG of isolates and virulence to tested hosts, though the pattern of correlation varied with the host. Thus, collectively, isolates of VCG2B and VCG4B were the most virulent and isolates of VCG1A or HSI were the least virulent to artichoke; however, isolates of VCG1A were the most virulent to cotton. Also, we found that subgrouping occurs among isolates in VCG2B based on genetic and molecular differences, and this subgrouping also correlated with virulence of isolates to the two hosts. This is the first genetic and virulence characterization of $V$. dahliae populations infecting artichoke in a large geographic area. Prior to this study, a single $V$. dahliae isolate from artichoke in California was assigned to VCG2 and it was highly virulent to both artichoke and cotton (5).

Many studies on VCG diversity in $V$. dahliae using isolates from diverse host sources and wide geographic origins have shown a worldwide distribution of VCG2 and VCG4 and the prevalence of VCG2B and VCG4B subgroups $(14,31,34,37,56)$. However, fewer studies of VCG diversity in major crops (mainly cotton, pepper, potato, and watermelon) in specific cropping areas $(3,25,33,39,50,52,53)$ showed one or two VCGs or VCG subgroups to predominate. For example, VCG2 (no indication was given regarding subgrouping) isolates prevails in pepper in California (3) and watermelon in Greece (25), VCG4 (mainly VCG4B) is common among isolates infecting potato in North America $(33,50,52,53)$, and VCG2B and VCG4B or VCG1 and VCG2A are common in cotton fields in Israel and Spain, respectively $(37,39)$. Our results agree with this limited VCG diversity occurring in a major crop, because $96 \%$ of $V$. dahliae isolates from artichoke in the Comunidad Valenciana in eastern-central Spain that could be typed to VCG were of VCG2, of which nearly $70 \%$ belonged to VCG2B. Furthermore, such limited VCG diversity shows a regional distribution within that area which resembled the regional distribution shown for VCG2B and VCG4B in cotton in Israel (37). Thus, although VCG2B was widespread across the artichoke-growing area, most isolates from the northern part of this area were of VCG2B and most isolates of VCG2A occurred in the southern part.

The reasons for a regional distribution of $V$. dahliae VCGs in artichoke in eastern-central Spain are not known. Artichoke crops in this area as well as other production regions in Spain are established using rooted stumps from harvested plants which are obtained mainly from artichoke crops in the Navarra region of northern Spain (Fig. 1). This suggests that $V$. dahliae might have been introduced into artichoke crops at the Comunidad Valenciana with infected planting material, because planting of infected stumps from symptomatic and asymptomatic plants in noninfested soil resulted in Verticillium wilt incidences that correlated with the incidence of stump infections (28). However, to the best of our knowledge, Verticillium wilt has not yet been recorded in artichoke crops at the Navarra region of Spain (J. Armengol and J. García-Jiménez, unpublished data). Alternatively, the regional distribution of $V$. dahliae VCGs in artichoke at the Comunidad Valenciana may reflect the cropping history of $V$. dahliaesusceptible plants in soils, although no clear relationship have been detected in the 18 wilt-affected artichoke fields with recorded cropping history. The occurrence of VCG1A infecting artichoke in the surveyed area was unexpected. The single
V. dahliae isolate V403 from artichoke in Valencia was assigned to VCG1A based on the vigor of heterokaryons formed between complementing nit mutants of this isolate and of reference VCG1 cotton isolates (presumably VCG1A) $(7,39)$ and non-cotton isolates (presumably VCG1B) $(7,15)$. In addition, isolate V403 was identified as $\mathrm{D}$ pathotype by the pathotype-specific molecular markers $(41,43)$ and defoliating disease reaction in 'Acala SJ-2' cotton $(8,39)$. VCG1A/D-pathotype is considered as a distinct subspecific population within $V$. dahliae indigenous to the southern and southwestern United States which now has spread to other geographic areas in America, Asia, China, Greece, Israel, and Spain $(8,21,26,38,40,58)$. Defoliating isolates of $V$. dahliae from China, Spain, and the United States appear molecularly similar and clonal, suggesting that they were introduced into those areas $(39,46)$. Worldwide spread of the D pathotype may have occurred with contaminated cotton seed (6). In Spain, the D pathotype was found restricted in cotton crops at the southern part of the country in the mid-1980s, but it now has spread beyond that area and causes severe defoliation in olive crops as well (41; R. M. Jiménez-Díaz, unpublished data). How the D pathotype became established in eastern-central Spain is not known. A small acreage of cotton (Gossypium hirsutum L.) was grown in the central and southern part of the Comunidad Valenciana, mainly in the 1950s, but cotton was never grown in the northern part of this region. To further explore the possibility of the $\mathrm{D}$ pathotype occurring in other locations of the surveyed area, we carried out a limited disease survey in cotton fields at Valencia and Alicante provinces. Verticillium wilt-affected cotton crops were found at a single location of the latter province, with $V$. dahliae isolates from only three fields at that location identified as VCG1A and D pathotype by the same procedures used to characterize isolates from artichoke (R. M. Jiménez-Díaz, unpublished data). It might be possible that $\mathrm{D}$ isolates were introduced in the Comunidad Valenciana long ago with contaminated seed of imported, improved cotton cultivars, but this pathotype had remained undetected until now.

The limited VCG diversity found among $V$. dahliae infecting artichoke in eastern-central Spain would suggest that genetic homogeneity might prevail in the predominant VCG2B. However, independent work by several authors in different geographic areas indicates that VCG2B is a rather heterogeneous group in physiological and morphological traits, as well as in host range and virulence to specific hosts $(34,37,39,56,57)$. In our study, differences both in the typing of isolates with reference strains of $V$. dahliae VCGs and in the amplification of DNA markers previously associated with the $\mathrm{D}$ and $\mathrm{ND}$ pathotypes indicated genetic heterogeneity in VCG2B isolates of $V$. dahliae infecting artichoke in the Comunidad Valenciana in eastern-central Spain. Conversely, VCG2A and VCG4B isolates of the pathogen from artichoke appeared homogeneous, based on consistent amplification of the 824-bp marker previously associated with ND $V$. dahliae (42), as well as in their compatibility with reference VCG tester strains. We do not have an explanation for the fact that some of VCG2B isolates (i.e., the VCG2Ba subgroup) failed to form stable heterokaryons with the reference VCG2B tester strains but did with nit mutants of isolates of the VCG2 Br subgroup that were identified with those reference testers. In any case, no crossreactions occurred between isolates of VCG2A and isolates of VCG2B. The tester strains used for the identification of VCG2B were cotton isolates from Israel that correlated with the OARDC reference strain 115 isolated from cotton in Syria $(37,49)$. It is conceivable that isolates from the same host in a geographic area may show closer relationships for heterokaryon compatibility compared with isolates from much different hosts and remote geographic locations. Also, it might be that artichoke isolates of VCG2Br are bridging isolates between VCG2Ba and the reference VCG tester isolates $(31,32)$. Bhat et al. (3) appear to have experienced a similar situation in the VCG characterization of $V$. dahliae isolates from pepper in California, whereby seven iso- 
lates did not form heterokaryons with any of the known VCG tester strains and were assigned to a new VCG, VCG6.

In addition to the differences in the relationships for heterokaryon compatibility, $V$. dahliae isolates of $\mathrm{VCG} 2 \mathrm{Ba}$ and VCG2Br also showed differences in the pattern of amplification of PCR-based markers previously associated with the cotton D and ND pathotypes. Thus, all VCG2Ba isolates yielded only the D-associated 334-bp amplicon in amplifications using primers DB19, DB22, and espdef01, but gave no amplification with primer pairs INTD2f/INTD2r or INTND2f/INTND2r which produce the 462- or 824-bp markers associated with the D and ND pathotypes, respectively (41-43). Conversely, isolates of the VCG2Br subgroup proved molecularly heterogeneous because, using the aforementioned primers, nearly $50 \%$ of them produced the 334-bp amplicon only and the other $50 \%$ yielded only the 824-bp marker. Our results agree with those of other authors who found that $V$. dahliae isolates within the same VCG either from the same or different hosts may show molecular variability, suggesting that those isolates derive from different clonal lineages $(3,23)$. In previous studies $(41)$, we found that the $V$. dahliae-specific PCR products amplified by primers DB19 and DB22 from cotton and olive D and ND isolates differed in a 15-nt insertion (that was lacking in ND isolates), and this insertion was the basis for designing the reverse primer espdef01 specific for $\mathrm{D} V$. dahliae. Our results suggest that the VCG1A/D-pathotype and some of the VCG2B isolates from artichoke might be molecularly related, though they differed in traits of biological significance such as virulence and the defoliation phenotype on artichoke and cotton. An important practical outcome from this present study is that the primers previously developed to distinguish between D and ND pathotypes eventually may not be reliable when used with $V$. dahliae isolates from hosts other that cotton and olive.

The results of virulence tests on artichoke and cotton cultivars showed small though statistically significant differences in virulence between $V$. dahliae isolates according to VCG, as well as in susceptibility between artichoke cultivars. That statistical significance of the small differences was because of low variability of the results in the experiments, as well as the high precision of the statistical analyses facilitated by the large set of data used (for example, 10 replicated plants for each of 35 isolates per artichoke cultivar). The small difference in susceptibility between the artichoke hybrid cvs. Num 6374 and Num 9444 is not of much biological significance; however, results indicate that both are very susceptible to Verticillium wilt. We chose to use these cultivars to avoid uncertain homogeneity of commercialized propagative artichoke stumps as well as to facilitate manipulation of plants for inoculation during experiments. The high susceptibility of the two artichoke cultivars may have masked larger differences in virulence between $V$. dahliae isolates according to VCG. These differences would likely be enlarged and, thus, their phytopathological significance enhanced, with less susceptible cultivars and environmental conditions less favorable for disease than those provided in our experiments, as shown by Korolev et al. (39) for $V$. dahliae VCGs from Israel on cotton cultivars. The correlation between VCG of artichoke V. dahliae isolates and virulence (i.e., the amount of disease caused in a host genotype) to artichoke cultivars found in this study corresponds with similar associations found by other authors for different hosts. For example, potato isolates of VCG4A in California are collectively more virulent to potato than those of VCG4B (2,53), and cotton isolates of VCG2B in Israel are considered to be, overall, more virulent to cotton than isolates of VCG4B, with the differences in virulence demonstrated under controlled conditions correlating with severe disease caused by VCG2B isolate in cotton fields (39). As a group, 21 VCG2B artichoke isolates were significantly more virulent to artichoke compared with $8 \mathrm{VCG} 2 \mathrm{~A}$ isolates tested; isolates of VCG4B were very virulent, too, but only 2 were tested. This result is of practical significance in terms of the use of disease prediction and resistant cultivars for the management of Verticillium wilt of artichoke; for instance, disease risk assessment of infested soils does not seem possible solely on the basis of quantitative estimation of inoculum in soil.

An additional aspect of the virulence tests in our study worth consideration concerns the stem-injection method used for inoculation of 'Acala SJ-2' cotton, compared with the root-dip inoculation of artichoke cultivars. The stem-injection method of inoculating cotton plants is easier to perform and more reproducible compared with the root-dip inoculation, and it does not cause the physiological stunting induced by root dipping, thus making it easier to determine the effect of infection on plant growth $(8,10,27,39)$. The stem-injection method commonly has been used in the research on Verticillium wilt of cotton for a long time, to determine both differences in virulence among $V$. dahliae isolates $(8,9,39,47)$ and tolerance to disease in cotton germ plasm. For example, Devey and Rosielle (22) found that Verticillium wilt reaction of cotton cultivars under field conditions and natural infection in $V$. dahliae-infested soil correlated with reaction of the same cultivars to stem-injection inoculation under controlled conditions, but not with reaction to inoculation by adding a conidial suspension of the pathogen around the root system of a potted plant. Similarly, Bejarano et al. (9) found that the difference in virulence between D and ND $V$. dahliae isolates from affected plants determined by the stem-injection method correlated with severity of Verticillium wilt development in cotton fields. Thus, for similar levels of inoculum of each of the pathotypes in soil, the epidemics caused by the more virulent $\mathrm{D}$ pathotype developed earlier and more rapidly and resulted in a higher final amount of disease compared with those caused by the less virulent ND pathotype. Therefore, the differences in virulence to cotton of $V$. dahliae isolates according to VCG found in the present study would be of use for prediction of severe Verticillium wilt in cotton crops.

Results of our virulence study differ from those of Bhat and Subbarao (5). Those authors found that a VCG2 isolate from artichoke was highly virulent both on artichoke and 'Acala SJ-2' cotton, and that a VCG1 cotton isolate was not pathogenic to artichoke. In our study, the VCG2 (VCG2A and VCG2B) artichoke isolates were more virulent to artichoke and less virulent to Acala SJ-2 cotton compared with virulence of VCG1A isolates from artichoke or cotton, respectively, to those same hosts. Differences in the isolates used, as well as in the inoculum density and inoculation method, together with the nature of the isolates, may account for the discrepancy. A lesser virulence of VCG1A/Dpathotype isolates to other hosts (such as eggplant and tomato) compared with that shown on cotton has been reported $(48,51)$.

In summary, the present study indicates that, although a limited VCG diversity may exist in a given crop at a given area, a degree of genetic heterogeneity still can occur within that which may be of significance for disease management. Further studies are needed for a deeper understanding of the nature of such heterogeneity. Studies are in progress to further assess the molecular diversity existing in $V$. dahliae VCGs infecting artichoke as well as other crop plants in different geographic locations in Spain, and to determine relationships that might exists among the prevailing VCGs.

\section{ACKNOWLEDGMENTS}

Research was supported in part by grants AGL 2000-1444 from Comisión Interministerial de Ciencia y Tecnología (CICYT) of Spain, and by grant GV-CAPA00-12 from Consellería de Agricultura, Pesca y Alimentación de Generalitat Valenciana. J. Mercado-Blanco is a contract holder under the 'Ramón y Cajal' Programme of the Spanish Ministry of Education and Science. We thank I. Susín from Nunhems Semillas, S. A. for supplying artichoke seed; P. Castillo, B. B. Landa, and J. A. NavasCortés for helpful comments on the manuscript prior to submission; and the senior editor and anonymous reviewers for suggestions and editorial improvement. 


\section{LITERATURE CITED}

1. Armengol, J., Berbegal, M., Giménez-Jaime, A., Romero, S., Beltrán, R., Vicent, A., Ortega, A., and García-Jiménez, J. 2005. Incidence of Verticillium wilt of artichokes in eastern Spain and role of inoculum sources on crop infection. Phytoparasitica 33:397-405.

2. Barbara, D. J., and Clewes, E. 2003. Plant-pathogenic Verticillium species: How many of them are there? Mol. Plant Pathol. 4:297-305.

3. Bhat, R. G., Smith, R. F., Koike, S. T., Wu, B. M., and Subbarao, K. V. 2003. Characterization of Verticillium dahliae isolates and wilt epidemics of pepper. Plant Dis. 87:789-797.

4. Bhat, R. G., and Subbarao, K. V. 1999. First report of Verticillium dahliae causing artichoke wilt in California. Plant Dis. 83:782.

5. Bhat, R. G., and Subbarao, K. V. 1999. Host range specificity in Verticillium dahliae. Phytopathology 89:1218-1225.

6. Bell, A. A. 1992. Verticillium wilt. Pages 87-126 in: Cotton Diseases. R. J. Hillocks, ed. CAB International, Wallingford, UK.

7. Bell, A. A. 1994. Mechanisms of disease resistance in Gossypium species and variation in Verticillium dahliae. Pages 225-235 in: Proc. World Cotton Res. Conf. 1. G. A. Constable and N. W. Forrester, eds. CSIRO, Melbourne, Australia.

8. Bejarano-Alcázar, J., Blanco-López, M. A., Melero-Vara, J. M., and Jiménez-Díaz, R. M. 1996. Etiology, importance, and distribution of Verticillium wilt of cotton in southern Spain. Plant Dis. 80:1233-1238.

9. Bejarano-Alcázar, J., Melero-Vara, J. M., Blanco-López, M. A., and Jiménez-Díaz, R. M. 1995. Influence of inoculum density of defoliating and nondefoliating pathotypes of Verticillium dahliae on epidemics of Verticillium wilt of cotton in southern Spain. Phytopathology 85:14741481.

10. Bugbee, W. E., and Presley, J. T. 1967. A rapid inoculation technique to evaluate the resistance of cotton to Verticillium albo-atrum. Phytopathology 57:1264.

11. Campbell, C. L., and Madden, L. V. 1990. Introduction to Plant Disease Epidemiology. John Wiley and Sons, New York.

12. Carder, J. H., Morton, A., Tabrett, A. M., and Barbara, D. J. 1994. Detection and differentiation by PCR of subspecific groups within two Verticillium species causing vascular wilts in herbaceous host. Pages 9197 in: Modern Assays for Plant Pathogenic Fungi: Identification, Detection and Quantification. A. Schots, F. M. Dewey, and R. Oliver, eds. CAB International, Oxford.

13. Chambonnett, D., Pochard, E., and Vigouroux, A. 1967. La verticilliose de L'Artichaut dans le Sud-Est de la France. Phytopathol. Mediterr. 6:9599.

14. Chandelier, A., Laurent, F., Dantinne; D., Mariage, L., Etienne, M, and Cavalier, M. 2003. Genetic and molecular characterization of Verticillium dahliae isolates from woody ornamentals in Belgian nurseries. Eur. J. Plant Pathol. 109:943-952.

15. Chen, W. 1994. Vegetative compatibility groups of Verticillium dahliae from ornamental woody plants. Phytopathology 84:214-219.

16. Ciccarese, F., Cirulli, M., and Frisullo, S. 1985. Prove di lotta chimica contro la Verticilliosi del carciofo. Inf. Fitopatol. 35(5):39-42.

17. Cirulli, M., Ciccarese, F., and Amenduni, M. 1994. Evaluation of Italian clones of artichoke for resistance to Verticillium dahliae. Plant Dis. 78:680-682.

18. Cirulli, M., Ciccarese, F., and Frisullo, S. 1984. L'avvizzimento del carciofo da Verticillium dahliae Kleb. in Italia meridionale. Inf. Agrario 40:52-55.

19. Collins, A., Mercado-Blanco, J., Jiménez-Díaz, R. M., Olivares, C., Clewes, E., and Barbara, D. J. 2005. Correlation of molecular markers and biological properties in Verticillium dahliae and the possible origins of some isolates. Plant Pathol. 54:549-557.

20. Correll, J. C., Klittich, C. J. R., and Leslie, J. F. 1987. Nitrate nonutilizing mutants of Fusarium oxysporum and their use in vegetative compatibility tests. Phytopathology 77:1640-1646.

21. Daayf, F., Nicole, M., and Geiger, J. 1995. Differentiation of Verticillium dahliae populations on the basis of vegetative compatibility and pathogenicity on cotton. Eur. J. Plant Pathol. 101:69-79.

22. Devey, M. E., and Rosielle, A. A. 1986. Relationship between field and greenhouse rating for tolerance to Verticillium wilt on cotton. Crop Sci. 26:1-4.

23. Dobinson, K. F., Harrington, M. A., Omer, M., and Rowe, R. C. 2000. Molecular characterization of vegetative compatibility group 4A and 4B isolates of Verticillium dahliae associated with potato early dying. Plant Dis. 84:1241-1245.

24. Dobinson, K. F., Paterson, N. A., White, G., and Grant, S. 1998. DNA fingerprinting and vegetative compatibility analysis indicate multiple origins for Verticillium dahliae race 2 tomato isolates from Ontario, Canada. Mycol. Res. 102:1089-1095.

25. Elena, K. 2000. Vegetative compatibility among Verticillium dahliae isolates from watermelon in Greece. Phytoparasitica 28:115-120.
26. Elena, K., and Paplomatas, E. J. 2001. The defoliating strain of Verticillium dahliae on cotton: First report for Greece. (Abstr.) Phytopathol. Mediterr. 40:70.

27. Erwin, D. C., Moje, W., and Malca, I. 1965. An assay of the severity of Verticillium wilt on cotton plants inoculated by stem puncture. Phytopathology 55:663-665.

28. Giménez-Jaime, A., Beltrán, R., Vicent, A., Armengol, J., and GarcíaJiménez, J. 2003. Differential infection of artichoke propagating material grown in Verticillium dahliae infested and noninfested soil. Acta Hortic. 660:501-505.

29. Gomez, K. A., and Gomez, A. A. 1984. Statistical Procedures for Agricultural Research, 2nd ed. John Wiley and Sons, New York.

30. Hastie, A. C., and Heale, J. B. 1984. Genetics of Verticillium. Phytopathol. Mediterr. 23:130-161.

31. Hiemstra, J. A., and Rataj-Guranowska, M. 2003. Vegetative compatibility groups in Verticillium dahliae isolates from the Netherlands as compared to VCG diversity in Europe and the USA. Eur. J. Plant Pathol. 109:827839 .

32. Joaquim, T. R., and Rowe, R. C. 1990. Reassessment of vegetative compatibility relationships among strains of Verticillium dahliae using nitrate-nonutilizing mutants. Phytopathology 80:1160-1166.

33. Joaquim, T. R., and Rowe, R. C. 1991. Vegetative compatibility and virulence of strains of Verticillium dahliae from soil and potato plants. Phytopathology 81:552-558.

34. Katan, T. 2000. Vegetative compatibility in populations of Verticillium- an overview. Pages77-94 in: Advances in Verticillium Research and Disease Management. E. Tjamos, R. C. Rowe, J. B. Heale, and D. Fravel, eds. The American Phytopathological Society, St. Paul, MN.

35. Klein, K. K., and Correll, J. C. 2001. Vegetative compatibility group diversity in Fusarium. Pages 83-96 in: Fusarium. Paul E. Nelson Memorial Symposium. B. A. Summerel, J. Leslie, D. Backhouse, W. L. Bryden, and L. W. Burgess, eds. The American Phytopathological Society, St. Paul, MN.

36. Korolev, N., and Katan, T. 1997. Improved medium for selecting nitrate nonutilizing (nit) mutants of Verticillium dahliae. Phytopathology 87: 1067-1070.

37. Korolev, N., Katan, J., and Katan, T. 2000. Vegetative compatibility groups of Verticillium dahliae in Israel: Their distribution and association with pathogenicity. Phytopathology 90:529-566.

38. Korolev, N., Katan, J., and Katan, T. 2000. Genetic variation and virulence of Verticillium dahliae from different hosts in Israel. Pages 280-281 in: Abstracts of Presentations. 21st Congress of the Israeli Phytopathological Society, Bet Dagan, Israel.

39. Korolev, N., Pérez-Artés, E., Bejarano-Alcázar, J., Rodríguez-Jurado, D., Katan, J., Katan, T., and Jiménez-Díaz, R. M. 2001. Comparative study of genetic diversity and pathogenicity among populations of Verticillium dahliae from cotton in Spain and Israel. Eur. J. Plant Pathol. 107:443-456.

40. Mathre, D. E., Edwin, D. C., Paulus, A. O., and Ravenscroft, A. V. 1966. Comparison of the virulence of isolates of Verticillium albo-atrum from several of the cotton-growing regions in the United States, Mexico and Peru. Plant Dis. Rep. 50:930-933.

41. Mercado-Blanco, J., Rodríguez-Jurado, D., Parrilla-Araujo, S., and Jiménez-Díaz, R. M. 2003. Simultaneous detection of the defoliating and nondefoliating Verticillium dahliae pathotypes in infected olive plants by duplex, nested polymerase chain reaction. Plant Dis. 87:1487-1494.

42. Mercado-Blanco, J., Rodríguez-Jurado, D., Pérez-Artés, E., and JiménezDíaz, R. M. 2001. Detection of the nondefoliating pathotype of Verticillium dahliae in infected olive plants by nested PCR. Plant Pathol. 50:609619.

43. Mercado-Blanco, J., Rodríguez-Jurado, D., Pérez-Artés, E., and JiménezDíaz, R. M. 2002. Detection of the defoliating pathotype of Verticillium dahliae in infected olive plants by nested PCR. Eur. J. Plant Pathol. 108:1-13.

44. Möller, E. N., Bahnwes, G., Sandermann, H., and Geiger, H. H. 1992. A simple and efficient protocol for isolation of high molecular weight DNA from filamentous fungi, fruit bodies, and infected plant tissues. Nucleic Acids Res. 20:6115-6116.

45. Pegg, G. F., and Brady, B. L. 2002. Verticillium Wilts. CAB International, Oxford.

46. Pérez-Artés, E., García-Pedrajas, M. D., Bejarano-Alcázar, J., and Jiménez-Díaz, R. M. 2000. Differentiation of cotton-defoliating and nondefoliating pathotypes of Verticillium dahliae by RAPD and specific PCR analyses. Eur. J. Plant Pathol. 106:507-517.

47. Pullman, G. S., and DeVay, J. E. 1982. Epidemiology of Verticillium wilt of cotton: A relationship between inoculum density and disease progression. Phytopathology 72:549-554.

48. Rodríguez-Morcillo, V., Bejarano-Alcázar, J., and Jiménez-Díaz, R. M. 2001. Host range of Verticillium dahliae pathotypes infecting cotton and olive crops in southern Spain. Page 72 in: Abstracts of Presentations. 8th Int. Verticillium Symp. Córdoba, Spain. 
49. Rowe, R. C. 1995. Recent progress in understanding relationships between Verticillium species and subspecific groups. Phytoparasitica 23:31-38.

50. Rowe, R. C., Johnson, D. A., and Omer, M. A. 2000. Vegetative compatibility analysis of strains of Verticillium dahliae from potato seed tubers and plants from western and eastern North America. Pages 95-99 in: Advances in Verticillium Research and Disease Management. E. Tjamos, R. C. Rowe, J. B. Heale, and D. Fravel, eds. The American Phytopathological Society, St. Paul, MN.

51. Schnathorst, W. C., and Mathre, D. E. 1966. Host range and differentiation of a severe form of Verticillium albo-atrum in cotton. Phytopathology 56:1155-1161.

52. Strausbaugh, C. A. 1993. Assessment of vegetative compatibility and virulence of Verticillium dahliae isolates from Idaho potatoes and tester strains. Phytopathology 83:1253-1258.

53. Strausbaugh, C. A., Schroth, M. N., Weinhold, A. R., and Hancock, J. G.
1992. Assessment of vegetative compatibility of Verticillium dahliae tester strains and isolates from California potatoes. Phytopathology 82:61-68.

54. Talboys, P. W. 1960. A culture medium aiding the identification of Verticillium albo-atrum and V. dahliae. Plant Pathol. 9:57-58.

55. Tjamos, E. C., and Paplomatas, E. J. 1988. Long-term effect of soil solarization in controlling Verticillium wilt of globe artichokes in Greece. Plant Pathol. 37:507-515.

56. Zeise, K., and von Tiedemann, A. 2001. Morphological and physiological differentiation of vegetative compatibility groups in Verticillium dahliae. J. Phytopathol. 149:469-475.

57. Zeise, K., and von Tiedemann, A. 2002. Host specialization among vegetative compatibility groups of Verticillium dahliae in relation to Verticillium longisporum. J. Phytopathol. 150:112-119.

58. Zhengjun, X., Achar, P. N., and Benkang, G. 1998. Vegetative compatibility groupings of Verticillium dahliae from cotton in mainland China. Eur. J. Plant Pathol. 104:871-876. 